\title{
On the cosmic-ray energy scale of the LOFAR radio telescope \\ ICRC 2021
}

K. Mulrey, ${ }^{1, *}$ S. Buitink, ${ }^{1,2}$ A. Corstanje, ${ }^{1,2}$ H. Falcke, ${ }^{2,3,4}$ B. M. Hare, ${ }^{5}$ J. R. Hörandel, ${ }^{1,2,3}$ T. Huege,,${ }^{1,6}$ G. K. Krampah, ${ }^{1}$ P. Mitra, ${ }^{1}$ A. Nelles, ${ }^{7,8}$ H. Pandya, ${ }^{1}$ J. P. Rachen, ${ }^{1}$ O. Scholten, ${ }^{9}$ S. ter Veen, ${ }^{2,4}$ S. Thoudam, ${ }^{10}$ T. N. G. Trinh $^{11}$ and T. Winchen ${ }^{12}$

${ }^{1}$ Astrophysical Institute, Vrije Universiteit Brussel, Pleinlaan 2, 1050 Brussels, Belgium

${ }^{2}$ Department of Astrophysics/IMAPP, Radboud University, P.O. Box 9010, 6500 GL Nijmegen, The Netherlands

${ }^{3}$ Nikhef, Science Park 105, 1098 XG Amsterdam, The Netherlands

${ }^{4}$ Netherlands Institute of Radio Astronomy (ASTRON), Postbus 2, 7990 AA Dwingeloo, The Netherlands

${ }^{5}$ University of Groningen, Kapteyn Astronomical Institute, Groningen, 9747 AD, Netherlands

${ }^{6}$ Institut für Kernphysik, Karlsruhe Institute of Technology (KIT), P.O. Box 3640, 76021, Karlsruhe, Germany

${ }^{7}$ DESY, Platanenallee 6, 15738 Zeuthen, Germany

${ }^{8}$ ECAP, Friedrich-Alexander-University Erlangen-Nürnberg, 91058 Erlangen, Germany

${ }^{9}$ Interuniversity Institute for High-Energy, Vrije Universiteit Brussel, Pleinlaan 2, 1050 Brussels, Belgium

${ }^{10}$ Department of Physics, Khalifa University, PO Box 127788, Abu Dhabi, United Arab Emirates

${ }^{11}$ Department of Physics, School of Education, Can Tho University Campus II, 3/2 Street, Ninh Kieu District, Can Tho City, Vietnam

${ }^{12}$ Max-Planck-Institut für Radioastronomie, Auf dem Hügel 69, 53121 Bonn

E-mail: kmulrey@vub.be

Cosmic rays are measured at LOFAR simultaneously with a dense array of antennas and with the LOFAR Radboud air shower Array (LORA) which consists of 20 scintillators. In this contribution we present cosmic-ray energy reconstruction using radio and particle techniques and discuss the event-by-event and absolute scale uncertainties. The energies reconstructed with each method are shown to be in good agreement. The radio-based reconstruction has smaller uncertainty on an event-to event basis, so LOFAR analyses will use that technique in the future. We also present the radiation energy of air showers measured at LOFAR. Radiation energy scales quadratically with the electromagnetic energy in an air shower, which can be related to the energy of the primary cosmic ray. Once the local magnetic field is accounted for, the radiation energy can be used to compare the energy measured at different locations using different techniques. We compare the LORA particle-based energy scale to that of the Pierre Auger Observatory and find that they agree to within $(6 \pm 20) \%$ for a radiation energy of $1 \mathrm{MeV}$. The uncertainty on the comparison is dominated by the antenna calibration of each experiment. We plan to reduce this uncertainty in the future using a portable radio array to cross-calibrate the energy scales of different experiments using radiation energy and the same antennas.

$37^{\text {th }}$ International Cosmic Ray Conference (ICRC 2021)

July 12th - 23rd, 2021

Online - Berlin, Germany

\footnotetext{
${ }^{*}$ Presenter
} 


\section{Introduction}

Accurately determining the energy of detected cosmic rays is critical for the interpretation of measured data. In this contribution we discuss how energy reconstruction is handled at the LOw Frequency ARray (LOFAR) radio telescope [1, 2]. As an air shower develops, broadband radio emission is generated, with the dominate contribution coming from the geomagnetically induced, time-varying transverse current that develops as the shower propagates [3, 4]. LOFAR measures this emission between 30 and $80 \mathrm{MHz}$. A scintillator array, the LOFAR Radboud Air Shower Array (LORA), also detects particles from the air shower that reach ground level and is used to trigger antenna readout [5].

Since each event is simultaneously sampled by the LOFAR antennas and the LORA scintillators, two separate energy reconstructions can be compared. These results have recently been published [6] and will be summarized here. We will discuss in more depth how the associated uncertainties are handled, as well as prospects for using radiation energy to compare the energy scales of different experiments.

\section{Energy reconstruction}

Each event detected at LOFAR is measured both with LOFAR antennas and LORA particle detectors. Until recently an absolute calibration for the radio measurements was unavailable. The radio data, which is highly sensitive to the development of the shower, was used to determine the event geometry, while the particle information was used to determine the energy for the event. An absolute radio calibration has since been developed [7], and so now separate radio and particle energy reconstructions are possible. Both reconstruction techniques are based on Monte Carlo simulations, where for each event, a set of simulations of iron and proton primaries is generated using CORSIKA (v7.7100) [8] with QGSJETII-04 [9] to provide particle information and CoREAS [10] to provide radio information. The simulated energy, $E_{\text {sim }}$, is determined using a preliminary fit to a $2 \mathrm{D}$ lateral distribution function [11].

In the case of the radio-based energy reconstruction, each simulated radio footprint is fit to the data using a $\chi^{2}$ fitting procedure, with the core position and an overall radio scale factor, $f_{r}$, for the signal strength as free parameters. The simulation with the lowest $\chi^{2}$ is taken to be the best simulation describing the event. $E_{\text {sim }}$ is multiplied by the best-fit $f_{r}$, which sets the radio-based energy for the event, as $E_{\text {radio }}=f_{r} \times E_{\text {sim }}$.

The particle-based energy reconstruction makes use of the best fit simulation as determined by the radio $\chi^{2}$ fit, and the core position from the radio measurement is also used. Again, a $\chi^{2}$ minimization is done which fits the simulated energy deposit to the measured deposit in the scintillators, now with a scale factor for the signal strength, $f_{p}$, as a free parameter. Multiplying $E_{\text {sim }}$ by this particle scale factor sets the particle-based energy for the event, as $E_{\text {part }}=f_{p} \times E_{\text {sim }}$. Because the particle-based reconstruction uses information about the geometry of the event derived from the radio fit, the two reconstructions are not entirely independent. However, the scale set for the particle-based energy is determined entirely by the scintillator data and calibration. We found that the energies reconstructed using the two techniques are consistent. More extensive details about the energy fitting procedures can be found in [6]. 


\section{Understanding the calibration and uncertainties}

Energy measurements are only meaningful if the detector is well calibrated and the uncertainties are understood. In this section, we give an overview of the calibration procedure for radio and particle measurements, and discuss how uncertainties are determined.

\subsection{Calibration}

Calibrating both the antennas that measure radio signals and the scintillators that measure energy deposits from particles requires comparing measured data to a known source. The LOFAR system response, including the antennas and signal chain, is calibrated using Galactic emission as a source, taking into account background noise due to electronics. This procedure results in a calibration with a systematic uncertainty of $13 \%$, which is dominated by the uncertainty on the underlying models used to predict the Galactic emission. This uncertainty propagates into the uncertainty on the energy. Details of the calibration procedure are given in [7].

The energy deposited by the shower particles in the scintillators is determined by calibrating the scintillators using single muons [5]. We build a distribution of energy deposits from single muons, both measured in the field and in the lab, from which the most probable value can be determined. Energy deposits from single muons are also simulated with GEANT4 [12], using a realistic description of the detector and arrival directions. This provides a distribution of simulated energy deposits from which we determine the most probable value. The peak of the measured muon distribution is compared to the simulated muon distribution peak, and we arrive at a calibration factor for the LORA scintillators. The resulting muon energy deposit distributions are shown in the left panel of Fig. 1. The simulated distribution is shown in red, muons measured in the field in blue, and muons measured in the lab in green.

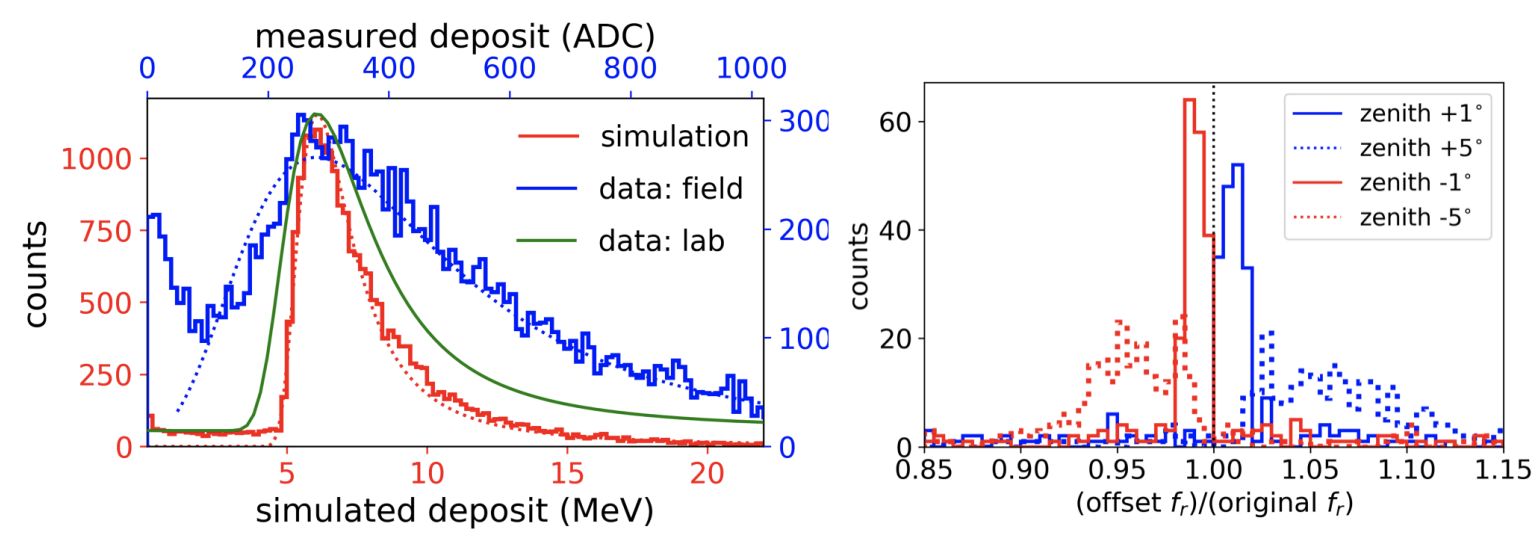

Figure 1: Left: Distributions of energy deposits in the LORA scintillators from single muons. The distribution in red shows the GEANT4 simulated deposits. The green and blue lines represent muon energy deposits measured in the lab and field, respectively. Right: The ratio between energy reconstructed with antenna model offsets and the original model. Offsets of $\pm 1^{\circ}$ and $\pm 5^{\circ}$ were applied.

There is a systematic uncertainty associated with the calibration of the LORA scintillators. By repeating the field calibration process a number of times, we find that the standard deviation of the 
calibration values for each scintillator is $\pm 3 \%$. This propagates into $\pm 3 \%$ uncertainty in event energy. The spatial response of the scintillators was measured at Karlsruhe Institute of Technology (KIT) and found to be very uniform over the surface of the scintillator. It is now included in LORA simulations [13].

\subsection{Event-by-event uncertainties}

Radio: angular dependence of the antenna model- We find the uncertainty in the angular dependence of the antenna model by offseting the antenna model by $\pm 1^{\circ}$ and $\pm 5^{\circ}$ in the zenith direction. Events are reprocessed with the offset antenna model. The ratio between the energy reconstructed with the offset and without the offset for each event is shown in Fig. 1. We conservatively take an uncertainty of $\pm 5 \%$, which is reflective of the distributions of the energy reconstruction ratio with the model offset $5^{\circ}$.

Radio and Particle: composition uncertainties- There is an uncertainty associated with the unknown primary composition of each event. The fact that the best fit simulation is associated with either a proton or an iron primary affects the reconstructed energy. In order to quantify this uncertainty, we ran simulations for a subset of events collecting a set of both 15 proton and 15 iron simulations with $X_{\max }$ values within $5 \mathrm{~g} / \mathrm{cm}^{2}$ of the reconstructed value of the event. We found that iron-initiated showers consistently reconstruct with an radio-based energy $10 \%$ higher than proton-initiated showers. For each event, we include an asymmetric 10\% uncertainty. Likewise, there is a composition uncertainty on the particle-based energy reconstruction. Given the same $X_{\max }$, proton-initiated showers reconstruct with a higher energy than iron-initiated showers. This effect is amplified with increasing zenith angle. We have parameterized the difference between proton and iron energy reconstruction as a function of zenith angle, and added an asymmetric error accordingly.

Particle: scintillator response variation- The response of the scintillators varies over time. We characterize the variation of each scintillator by looking at the daily average energy deposit. The variation is then determined by taking the standard deviation of average energy deposits. We propagate this uncertainty into the energy reconstruction, This process was repeated three times, and the maximum effect on the resulting energy scale was $2.5 \%$.

Radio and Particle: reconstruction uncertainties- The reconstruction uncertainty of each event is derived using a Monte Carlo vs. Monte Carlo method. The simulation set created for each event contains at least 40 showers. One shower is used as mock "data," and noise is added that reflects the noise level in the actual event data. This "event" is then reconstructed using the remaining simulations. The fit produces the scale factor $f_{r}$, used to convert the simulated energy $E_{\text {sim }}$ to event energy as $E_{\text {radio }}=f_{r} \times E_{\text {sim. }}$. Each shower in the set is simulated at the same energy, so if the fit were perfect the scale factor would always be $f_{r}=1$. This procedure is repeated using every simulation in the set as the "event", yielding a set of scale factors, $f_{r}$. The same procedure is also applied to the particle data, yielding a set of reconstructed scale factors $f_{p}$. The resulting distributions of radio and particle scale factors for one representative event are shown in the inset histograms in Fig. 2. One standard deviation of the distribution is taken to be the fit uncertainty for a particular event. The large histograms in Fig. 2 show the distribution of the standard deviation of scale factors for each event, or equivalently, the reconstruction uncertainty, for all events. Typical values for reconstruction uncertainties on radio-based energy are close to $9 \%$ with little spread. The 

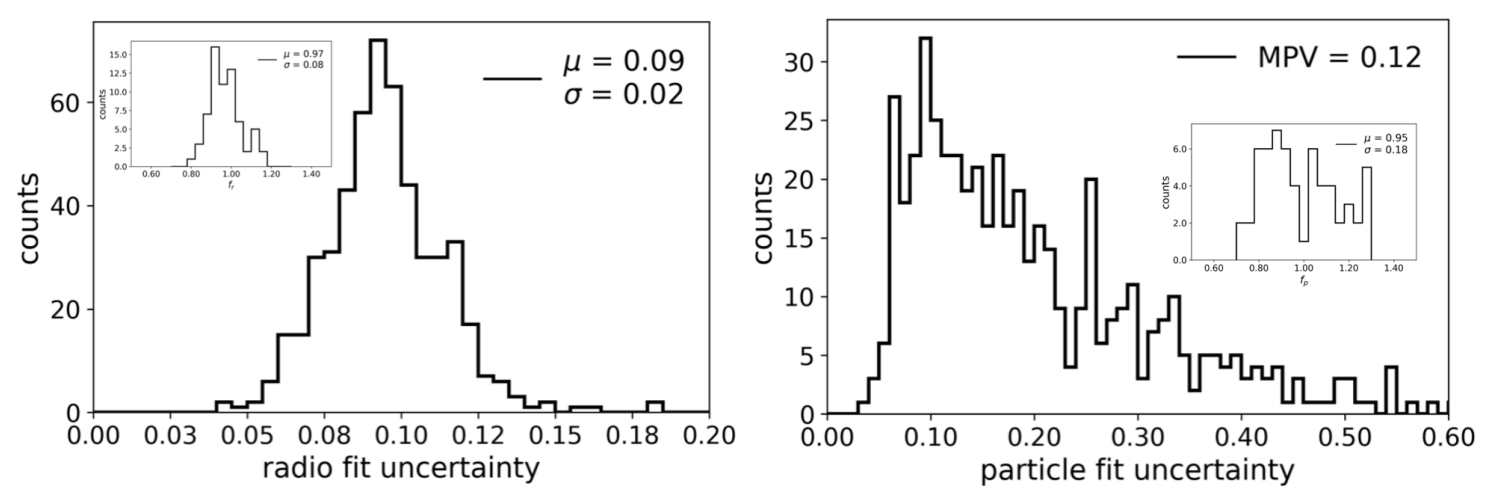

Figure 2: Inset histogram: resulting distributions of scale factors for one representative event. Main histogram:distribution of the standard deviation of scale factors for each event, or equivalently, the reconstruction uncertainty, for all events. Left: radio-based reconstruction. Right: particle-based reconstruction.

most probable value (MPV) of the particle-based uncertainties is $12 \%$, but they have a much larger spread and extend past $50 \%$.

\subsection{Systematic Uncertainties}

Radio: choice of simulation code-The simulated radio emission for this analysis was generated using CoREAS, however, there are other options, such as ZHAireS [14]. The agreement between the two simulation codes was studied in [15], and the effect of choosing one code over the other on the energy scale was determined to be less than $2.6 \%$.

Radio and particle: hadronic interaction model- In order to determine the effect of the choice of hadronic interaction model used in the CORSIKA simulation, ten events were re-analyzed using simulations produced with the Sibyll 2.3c [16] interaction model instead of QGSJETII-04. This changed the resulting radio-based energy by $3 \%$ and particle-based energy by $7 \%$.

\subsection{Conclusion}

The radio-based event-to-event uncertainties are a combined $11 \% \bigoplus$ reconstruction uncertainties. This is typically around $14 \%$ total. The radio-based systematic uncertainties (including calibration) are $13.6 \%$. The particle-based event-to-event uncertainties are a combined $2.5 \% \bigoplus$ composition uncertainty $\bigoplus$ reconstruction uncertainties, which vary widely. The total can extend from $10 \%$ up to $50 \%$. The particle-based systematic uncertainties (including calibration) total $7.6 \%$. In the past, the particle measurements have been used to set the LOFAR energy scale. Now, with an absolute radio calibration, and because the radio-based event-to-event uncertainties are significantly smaller, LOFAR has moved to using the radio measurements to set the energy scale.

\section{Comparing the energy scales of different experiments}

Radiation energy, $S_{R D}$, the total amount of radio emission emitted by an air shower, can be used to compare the energy scales of different experiments when measured together with the total shower energy determined using an independent method. The radiation energy contained in an air shower can be found by integrating the energy fluence over the radio footprint. 


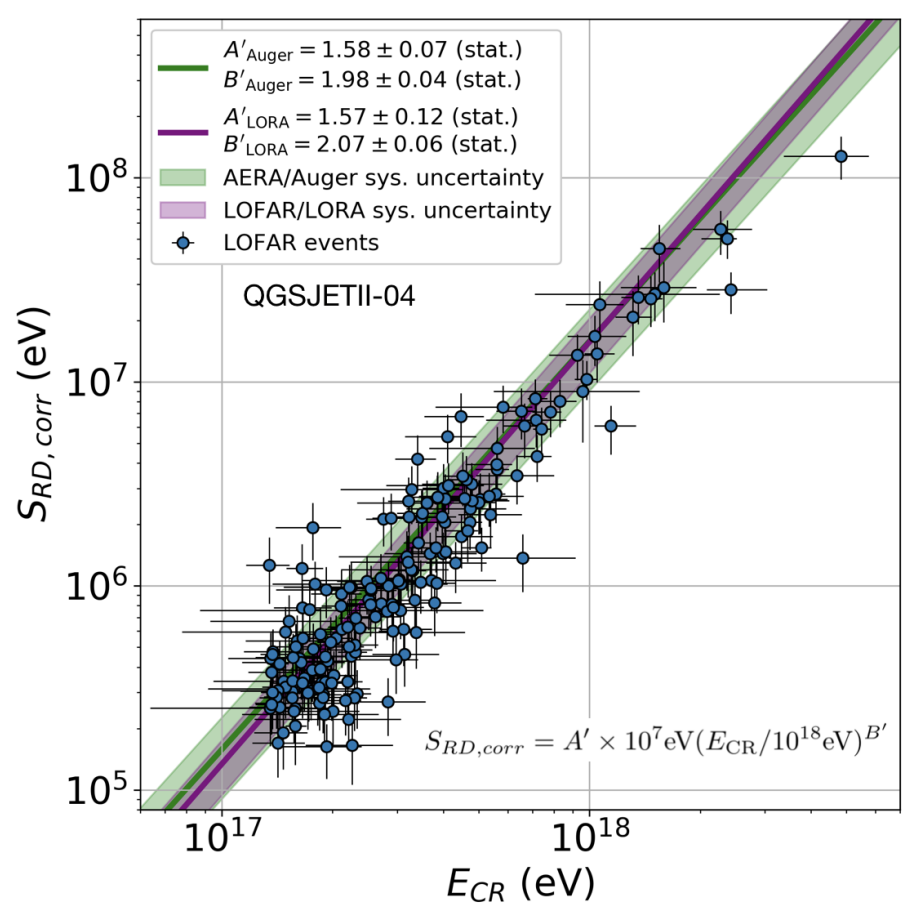

Figure 3: Relation between the corrected radiation energy measured by the LOFAR antennas and the cosmic-ray energy measured by the LORA scintillators. The error bars represent eventby-event uncertainties. The purple line shows the best fit line for LOFAR measurements of $S_{R D, c o r r}$. and LORA cosmic-ray energy, and the banded region around the best fit line represents the systematic uncertainties on the corrected radiation energy. The green line is the best fit line for AERA measurements of $S_{R D, c o r r}$. and Auger cosmicray energy [19], and the shaded green region represents the systematic uncertainties on the corrected radiation energy.

Radio waves are not attenuated in the atmosphere, and so the measurement is not affected by the location of the experiment (as long as all the radiation energy in the shower has been released before it reaches the ground). Some adjustments can be made to radiation energy measurements to make it a universal quantity, as discussed in [17]. The strength of the local magnetic field influences the strength of the geomagnetic emission. This can be normalized to the magnetic field of a reference location. Furthermore the geomagnetic contribution to the radiation energy can be adjusted to account for the angle between the magnetic field and shower axis. Making these corrections (and other second order corrections as in [17]) yields the "corrected radiation energy," $S_{R D, c o r r}$. We have found $S_{R D, \text { corr }}$ for LOFAR events, and found a relation between it and the total cosmic-ray energy, $E_{C R}$, as determined by LORA. A line of the form

$$
S_{R D, \text { corr }}=A^{\prime} \times 10^{7} \mathrm{eV}\left(E_{C R} / 10^{18} \mathrm{eV}\right)^{B^{\prime}}
$$

describes the relation well and is shown in Fig. 3. (The prime notation is consistent with what is written in [6].) The purple line is the best fit line of the form of Eq. 1 to the data. The shaded purple region represents the systematic uncertainty. More details about LOFAR $S_{R D, \text { corr }}$ are found in [6].

We compare the energy scales of LORA and Auger [18] by making use of the relation between the $S_{R D \text {, corr }}$ measured with the Auger Engineering Radio Array (AERA) and $E_{\mathrm{CR}}$, as determined by the Auger surface detectors, which are in turn calibrated using the fluorescence detectors [19]. The resulting relation, also of the form of Eq. 1, is show as the green line in Fig. 3. The shaded green region represents the systematic uncertainties on the corrected radiation energy.

We compare the energies of LORA and Auger at $S_{R D, \text { corr }}=1 \mathrm{MeV}$ and find ratio between the two to be $E_{\mathrm{CR}}{ }^{\text {LORA }} / E_{\mathrm{CR}^{\text {Auger }}}=1.06 \pm 0.20$. The uncertainty on the comparison includes only the radio-based uncertainties on the radiation energy, which is dominated by the calibration of the respective antennas. The energy scales of Auger and LORA agree within the comparison 
uncertainty, although the comparison uncertainty is larger than the absolute uncertainty on either energy scale.

Having a method to compare the energy scales of different experiments with minimal uncertainty is necessary in order to make meaningful comparisons of their spectra and composition measurements. In fact, energy scales have already been compared using radio techniques, although not using radiation energy [20]. We plan to use radiation energy and the same detection system to build a universal energy scale. A portable array of antennas will be built and deployed at different experiments, measuring radiation energy in conjunction with the host experiments' traditional air shower measurements. Using radiation energy to compare the energy scales eliminates uncertainties due to measurements being made at different locations, and using the same array eliminates the uncertainties associated with the antennas and calibration. This will allow for a cross-calibration of the energy scales of different experiments with minimal uncertainty [21].

\section{Summary}

We have discussed the radio and particle-based energy reconstruction techniques for LOFAR antenna measurements and LORA particle measurements respectively. We have described how event-by-event and systematic uncertainties are determined, and concluded that LOFAR analyses will use the radio-based energy reconstruction in the future. Minimizing uncertainties on the radiobased reconstruction is especially important when using radiation energy to compare the energy scales of different experiments. Since the antenna calibration is the dominant systematic uncertainty in radio-based reconstruction, we plan an experiment to cross-calibrate the energy scales of different experiments using radiation energy and the same antennas, thereby limiting systematic uncertainties on the comparison [21].

\section{References}

[1] M. P. van Haarlem et al. LOFAR: The LOw-Frequency ARray. Astronomy and Astrophysics, 556:56, 2013.

[2] P. Schellart et al. Detecting cosmic rays with the LOFAR radio telescope. Astronomy and Astrophysics, 560(A98), 2013.

[3] K. Werner and O. Scholten. Macroscopic Treatment of Radio Emission from Cosmic Ray Air Showers based on Shower Simulations. Astropart. Phys., 29:393-411, 2008.

[4] H. Falcke et al. Detection and imaging of atmospheric radio flashes from cosmic ray air showers. Nature, 435:313-316, 2005.

[5] S. Thoudam et al. LORA: A scintillator array for LOFAR to measure extensive air showers. Nucl.Instrum.Meth, A767:339-346, 2014.

[6] K. Mulrey et al. On the cosmic-ray energy scale of the LOFAR radio telescope. JCAP, 11:017, 2020. 
[7] K. Mulrey et al. Calibration of the LOFAR low-band antennas using the Galaxy and a model of the signal chain. Astropart. Phys., 111:1-11, 2019.

[8] D. Heck et al. CORSIKA: A Monte Carlo code to simulate extensive air showers. Report FZKA, 6019, 1998.

[9] S. Ostapchenko. Monte Carlo treatment of hadronic interactions in enhanced Pomeron scheme: QGSJET-II model. Phys. Rev. D, 83(014018), 2011.

[10] T. Huege, M. Ludwig, and C. W. James. Simulating radio emission from air showers with CoREAS. AIP Conf. Proc., 1535:128, 2013.

[11] A. Nelles et al. A parameterization for the radio emission of air showers as predicted by CoREAS simulations and applied to LOFAR measurements. Astropart.Phys., 60:13-24, 2015.

[12] S. Agostinelli et al. GEANT4 -a simulation toolkit. NIMPA, 506(3):250-303, 2003.

[13] K. Mulrey. Extension of the LOFAR Radboud Air Shower Array. In Proceedings, 36th International Cosmic Ray Conference (ICRC2016): Madison, Wisconson, July 24-August 1, 2019, page 363, 2019.

[14] J. Alvarez-Muniz, A. Romero-Wolf, and E. Zas. Cherenkov radio pulses from electromagnetic showers in the time-domain. Phys. Rev., D81:123009, 2010.

[15] M. Gottowik, C. Glaser, T. Huege, and J. Rautenberg. Determination of the absolute energy scale of extensive air showers via radio emission: systematic uncertainty of underlying firstprinciple calculations. Astropart. Phys., 103:87-93, 2018.

[16] R. S. Fletcher, T. K. Gaisser, P. Lipari, and T. Stanev. SIBYLL: An Event generator for simulation of high-energy cosmic ray cascades. Phys. Rev., D50:5710-5731, 1994.

[17] C. Glaser, M. Erdmann, J. R. Hörandel, T. Huege, and J. Schulz. Simulation of Radiation Energy Release in Air Showers. JCAP, 1609(09):024, 2016.

[18] J. Abraham et al. The Pierre Auger Cosmic Ray Observatory. Nuclear Instruments and Methods in Physics Research Section A: Accelerators, Spectrometers, Detectors and Associated Equipment, 798:172 - 213, 2015.

[19] A. Aab et al. Measurement of the Radiation Energy in the Radio Signal of Extensive Air Showers as a Universal Estimator of Cosmic-Ray Energy. Phys. Rev. Lett., 116(24):241101, 2016.

[20] W.D. Apel et al. A comparison of the cosmic-ray energy scales of Tunka-133 and KASCADEGrande via their radio extensions Tunka-Rex and LOPES. Phys. Lett. B, 763:179-185, 2016.

[21] K. Mulrey. Cross-calibrating the energy scales of cosmic-ray experiments using a portable radio array. In Proceedings, 36th International Cosmic Ray Conference (ICRC2021): Berlin, 2021, 2021. 